Gudrun Bucher (2009): Russisch-Amerika in deutschen Museen. In: Elfriede Hermann, Karin Klenke und Michael Dickhardt (Hg.): Form, Macht, Differenz. Motive und Felder ethnologischen Forschens. Göttingen: Universitätsverlag Göttingen, 149-161. Doi: 10.17875/gup2020-1281

\title{
Russisch-Amerika in deutschen Museen
}

\author{
Gudrun Bucher
}

Aber die Artefakte sind nur vermeintlich die gleichen geblieben; geändert hat
sich unser Blick, die Fragen, die wir an diese Objekte und ibre Herkunft richten,
und schließlich die Aussagen, von denen wir meinen, daß wir sie ihnen ent-
nehmen können. So verändert sich unsere Sichtweise mit jeder neuen Epoche,
und auch für die Angehörigen der Kulturen, aus denen sie stammen, hat sich
die Bedeutung der Objekte gewandelt. Die Gegenstände als solche sind deshalb
nicht Gegenstände an sich, die wir wahrnehmen, ist doch die Wabrnehmung ab-
hängig nicht nur vom Individuum, sondern auch von der Zeit und der Kultur,
in der es lebt. Auf diese Weise,verändern'sich kontinuierlich auch die Objekte.

(Hauser-Schäublin 1998:11)

Unter Russisch-Amerika versteht man jene Küsten und Inseln Alaskas, die von ihrer Entdeckung 1741 bis zum Verkauf an die USA im Jahre 1867 unter russischer Verwaltung standen. An der Erforschung dieses Gebietes waren häufig auch deutschsprachige Gelehrte beteiligt, wie z. B. Georg Wilhelm Steller, Carl Heinrich Merck, der Schweizer Johann Kaspar Horner, Georg Heinrich von Langsdorff, Adalbert von Chamisso und andere mehr. Im Rahmen der sogenannten Zweiten Kamtschatkaexpedition, die sowohl vom russischen Zarenhaus als auch von der russischen Akademie der Wissenschaften gefördert wurde, wurde im Jahr 1741 der Seeweg zur Inselkette der Aleuten entdeckt, was dazu führte, dass russische Pelzhändler ihr Augenmerk auf die neu entdeckte Region im weit entfernten Osten legten. War bislang der Zobel das Ziel ihrer Interessen, traten nun der Seeotter und die Pelzrobbe in den Vordergrund. Zunächst wurden die auf eigene Rechnung agierenden „promyšlenniki“ (Jäger, Pelzhändler, Abenteurer in 
einer Person) aktiv. Katharina II. formulierte gleich nach ihrer Thronbesteigung 1762 staatliches Interesse an der Region im fernen Osten, indem sie der Marine befahl, die bis dahin ausschließlich auf privater Basis operierenden „promyšlenniki“ zu unterstützen. In der zweiten Hälfte des 18. Jahrhunderts wuchs das Interesse an der Region des nördlichen Pazifiks auch in Europa und Amerika. Englische, französische und spanische Expeditionen suchten die Gewässer zwischen Russland und Alaska auf: so erhielt auch Kapitän James Cook 1778 für seine dritte Reise den Auftrag, die Nordwestküste Amerikas zu erkunden, durch die Beringstraße zu segeln und nach der Nordwestpassage zu suchen. Ausgelöst durch Cooks Aufenthalt im Nordpazifik wuchs das internationale Interesse am üppigen Vorkommen von Seeottern und Pelzrobben in der Region. Da Katharina II. befürchtete, ausländische Mächte könnten Gebietsansprüche erheben, sah sie sich gezwungen, Präsenz zu zeigen: Sie gab die „Geographisch-Astronomische Expedition in den nordöstlichen Teil von Russland“ (1785-1792) in Auftrag und übertrug die Leitung Joseph Billings. Billings stand zu diesem Zeitpunkt in russischen Diensten, hatte die Gegend aber bereits als Teilnehmer an Cooks dritter Reise kennen gelernt. Durch diese Expedition wurden die Aleuten und die Insel Kodiak endgültig der russischen Krone unterstellt und der Anspruch Russlands auf die amerikanischen Besitzungen wurde international gefestigt. Billings sammelte in staatlichem Auftrag ethnographische Realien (siehe unten).

Im Jahr 1799 schließlich gewährte Zar Paul I. der Pelzhandelskompanie ŠelichovGolikov das lang ersehnte Monopol und es kam zur Gründung der sogenannten Russisch-Amerikanischen Kompanie (RAK), die in der Folge mit der Verwaltung der Kolonie betraut wurde. Es handelte sich dabei um eine von der Regierung kontrollierte Aktiengesellschaft (Amburger 1966:368). Der jeweilige Gouverneur war Repräsentant der russischen Krone und die Handelsgesellschaft war für das Gesundheits- und Schulwesen sowie für die russisch-orthodoxe Mission verantwortlich (vgl. Black 2004, Luehrmann 2008).

Die nach Deutschland gelangten ethnographischen Sammlungen aus RussischAmerika, um die es im Folgenden gehen soll, gewähren einen Einblick in weltweit bestehende Beziehungsgeflechte während der zweiten Hälfte des 18. Jahrhunderts und der ersten Hälfte des 19. Jahrhunderts. Sie sind damit auch als Dokumente globaler Verflechtungen zu lesen, denn dynastische Verbindungen, Verwandtschafts-, Freundschaftsbeziehungen oder andere Verbindlichkeiten führten dazu, dass Gebrauchsgegenstände der indigenen Bevölkerung Russisch-Amerikas - Aleuten, Alutiiq, seltener Yupik oder Inupiaq aber auch von indianischen Gruppen der Nordwestküste - in verschiedene Museen Europas gelangten. Ein wachsendes wissenschaftliches Interesse an der Natur und an fernen Gegenden führte vermehrt zur Gründung von Schausammlungen für ein öffentliches Publikum. Mit der Einführung des neuen taxonomischen Systems für die Naturkunde wurde eine konzeptuelle Trennung zwischen „Kunst“ und „Natur“ vollzogen. Es wurden vermehrt reine Naturalienkabinette angelegt, für die systematisch gesammelt werden musste. Gingen Naturforscher auf weite Reisen, wurden sie 
beauftragt, gezielt zu sammeln, und wenn sie schon einmal unterwegs waren, legten sie „nebenher" auch Sammlungen von Ethnographica an. Zwar gab es kein taxonomisches System für Kulturen, aber meist versuchten sie, charakteristische Beispiele des materiellen Besitzes der jeweiligen Ethnien zu erwerben (Feest 1993:6). Häufig wurde Fremdheit, d.h. größtmögliche Verschiedenheit zum Eigenen - also die Differenz zum Kriterium der Auswahl von Objekten. Neben der Differenz spielen auch Form und Macht eine zentrale Rolle beim Erwerb und späteren Umgang mit Sammlungen. Mit den Reisen von James Cook zeichnete sich ein Paradigmenwechsel im Umgang mit Gegenständen fremder Kulturen ab: „Forms no longer were measured purely by comparison to similar forms in Europe. Differences were appreciated and explained in terms of their function in the other culture" (Feest 1993:6). Gesammelt wurden die Objekte jeweils von Repräsentanten der russischen Krone, die Macht über die indigene Bevölkerung ausübte und ihre Arbeitskraft zum Teil brutal ausbeutete (vgl. Luehrmann 2008). In manchen Fällen stammten sie auch von mehr oder weniger wissenschaftlich ausgerichteten Expeditionen, die ebenfalls im Auftrag der fremden Herrscher stattfanden.

Zwei der ältesten dieser Sammlungen befinden sich in der Ethnologischen Sammlung der Universität Göttingen. Es handelt sich dabei um Bestände aus den Sammlungen James Cooks und des Baron Georg Thomas von Asch. Beide Sammlungen wurden auf Initiative von Brigitta Hauser-Schäublin in umfangreich illustrierten Publikationen der Öffentlichkeit zugänglich gemacht (Hauser-Schäublin und Krüger 1998, 2007).

\section{Sammlung Cook/Forster Ethnologische Sammlung der Universität Göttingen}

$\mathrm{Ab}$ den frühen 1770er Jahren gelangten auf unterschiedliche Art und Weise völkerkundliche Objekte, die von Cooks Reisen stammten, nach Göttingen (Urban 1998:57). Der Hauptanteil der Bestände kam in Form einer königlichen Schenkung nach Göttingen. Johann Friedrich Blumenbach hatte, als er selbst für die Sammlungen verantwortlich war, im August 1781 eine Anfrage an die „Herren Geheimen Räthe“ in Hannover gerichtet, die diese im Oktober desselben Jahres an König Georg III. in London weiterleiteten. Bereits im folgenden Jahr konnte Blumenbach das Geschenk in Empfang nehmen. Einige Objekte wurden noch etwas später nachgeschickt (Urban 1998:59f.). Als Bestandteil dieser Sendungen gelangten auch einige Objekte aus Russisch-Amerika bzw. der Amerikanischen Nordwestküste nach Göttingen. Es handelt sich um acht Gegenstände der Nootka (NW-Küste) und um sechs Objekte der Eskimo und Aleuten, im einzelnen ein Bogen, ein Pfeilschaft, zwei Speerschleudern und zwei Knochenschnitzereien. 


\section{Sammlung Asch \\ Ethnologische Sammlung der Universität Göttingen}

Georg Thomas von Asch wurde 1729 in St. Petersburg geboren und dort im Wesentlichen von Hauslehrern erzogen. Sein Medizinstudium absolvierte er in Deutschland an den Universitäten Tübingen (1744-1747) und Göttingen (1748-1750). Nach Abschluss seiner Promotion in Göttingen kehrte von Asch nach St. Petersburg zurück, wo er in den russischen Staatsdienst eintrat und bis zum Generalstabsarzt aufstieg. Ab 1771 begann von Asch nach damaligem Wissenschaftsverständnis bedeutsames Material an die Göttinger Universität zu schicken. Zunächst trafen seine Sendungen nur sporadisch ein und enthielten vor allem islamische, religiöse Schriften, aber nachdem er im Oktober 1777 zum Auswärtigen Mitglied der Göttinger Gesellschaft der Wissenschaften ernannt worden war, schickte von Asch regelmäßig Pakete nach Göttingen. Seine Sendungen beinhalteten Bücher, Handschriften, Münzen und Medaillen; es waren aber auch Landkarten und Zeichnungen sowie Mineralien, Fossilien, Pflanzensamen und menschliche Skelette, insbesondere Schädel, darunter. Dank seines ausgedehnten Korrespondenznetzes und seiner guten Kontakte zu Kollegen und Gelehrten in ganz Russland gelang es ihm ebenfalls, Gebrauchsgegenstände verschiedener Ethnien des Russischen Reiches und angrenzender Gebiete zu erwerben und diese nach Göttingen zu transferieren. Darunter sind insgesamt 65 Objekte aus Russisch-Amerika, ein Großteil davon stammt von der Billings-Expedition (vgl. Bucher 2007:243-252).

\section{Sammlungen Krusenstern/Langsdorff und Leuchtenberg Staatliches Museum für Völkerkunde, München}

Die Versorgung von Russisch-Amerika mit Gebrauchsgütern und Getreide blieb bis zum Verkauf Alaskas im Jahr 1867 ein nicht zu unterschätzendes Problem. Um den langen und mühsamen, zum Teil auch gefährlichen Landweg zu umgehen, begann Russland zu Beginn des 19. Jahrhunderts, regelmäßig Weltumsegelungen durchzuführen. Die erste fand in den Jahren 1803-1806 statt und wurde von den Kapitänen Adam Johann von Krusenstern und Jurij Fedorovič Lisianskij geleitet. Als Gelehrter reiste neben anderen Georg Heinrich von Langsdorff mit. Er hatte in den Jahren 1793-1797 bei Johann Friedrich Blumenbach in Göttingen studiert und den Doktorgrad in Medizin erworben. Langsdorff brachte von den Aleuten und den Bewohnern der Insel Kodiak Objekte mit nach Europa und schenkte sie im Jahr 1823 dem König Maximilian I. Joseph von Bayern. 1868 gelangten sie in die „Königlichen Ethnographischen Sammlungen" und heute sind sie Bestandteil des Staatlichen Museums für Völkerkunde in München, wo sie als Sammlung Krusenstern inventarisiert sind (Rousselot 1994:25). Es handelt sich dabei um insgesamt 187 Nummern, die 113 Objekte aus Russisch-Amerika beinhalten (Rousselot 2007:74). Langsdorff hatte der Sammlung ein Verzeichnis 
beigegeben, in dem die Objekte auch beschrieben waren. Rousselot zufolge ist dieses Verzeichnis heute leider nicht mehr auffindbar. Einen Hinweis auf die Existenz dieses Verzeichnisses liefert aber der Münchener Akzessionskatalog, in dem sich Zitate daraus befinden.

Eine weitere Sammlung im Altbestand des Museums für Völkerkunde, München umfasst 35 Objekte aus dem Insel- und Küstengebiet des Nordpazifiks und stammt aus dem Besitz der Familie Leuchtenberg. Von dieser Sammlung sind weder der eigentliche Sammler noch die Umstände ihrer Entstehung bekannt (Rousselot 2007:75). Rousselot zufolge lassen sich aber zwei Theorien darüber aufstellen, wie die Objekte nach München gelangt sind. Der einen Variante zufolge gehen die Objekte auf Maximilian, den dritten Herzog von Leuchtenberg (1817-1852) zurück. Er lebte in Russland und vermählte sich 1839 mit Maria Nikolaevna, der ältesten Tochter von Zar Nikolaus I. Es wäre denkbar, dass er die Objekte in St. Petersburg erworben und sie seinem Vater, dem ersten Herzog von Leuchtenberg in Eichstätt geschenkt hat. Als sich nämlich die Familie Leuchtenberg 1858 endgültig in Rußland niederließ und das Fürstentum Eichstätt durch Rückkauf wieder Teil des Bayerischen Staates wurde, gehörte auch eine Sammlung von Ethnographica zu den erworbenen Gütern (Rousselot 2007:75). Die Objekte könnten aber auch aus dem Besitz von Joséphine de Beauharnais, der Mutter des ersten Herzogs von Leuchtenberg und ersten Frau Napoleons stammen, da verbürgt ist, dass auch sie eine ethnographische Sammlung besaß und nicht geklärt ist, was damit nach ihrem Tod geschehen ist (Rousselot 2007:76).

\section{Sammlung von Wrangell bzw. von Rossillon Museum der Weltkulturen, Frankfurt a.M.}

Die Jahre 1831 bis 1845 waren für die Russisch-Amerika Bestände europäischer Museen besonders fruchtbar. Drei Gouverneure, die nacheinander ihren Dienst versahen, legten umfangreiche Sammlungen an, und verfügten über entsprechende Verbindungen, Teile davon ins Ausland zu schicken. Im Einzelnen handelt es sich um Ferdinand von Wrangell (Gouverneur von 1831-1836), Ivan Antonovič Kuprejanov (Gouverneur von 1836-1840) und Arvid Adolf Etholén (Gouverneur von 1840-1845). Alle drei waren bereits vor ihrer jeweiligen Gouverneurszeit in Russisch-Amerika gewesen und hatten schon während dieser früheren Reisen begonnen zu sammeln.

Ferdinand Petrovič von Wrangell wurde am 29. Dezember 1796 in Estland geboren. Nach dem Besuch der Kadettenschule sammelte er erste Erfahrungen während der Weltumsegelung unter Kapitän V. M. Golovnin 1817-1819. In den Jahren 1820-24 unternahm von Wrangell eine Expedition in Nordost Sibirien. Seine zweite Weltumsegelung kommandierte er bereits selbst auf der Brigg Krotkii. Vom 21. September bis 12. Oktober 1826 hielt er sich in Sitka auf und war am 14. September 1827 wieder zurück in Kronstadt. Bald nach seiner Rückkehr erhielt er die Berufung zum Gouver- 
neur von Russisch-Amerika und machte sich erneut auf in den „Fernen Osten“, wo er von 1831 bis 1836 sein Amt ausübte und damit der direkte Vorgänger von Kuprejanov war (Pierce 1990).

Die Sammlung von Wrangell gelangte bereits 1834, also noch während Wrangells Dienstzeit in Russisch-Amerika, nach Frankfurt a. M. Sein Schwiegervater, Baron Wilhelm von Rossillon, schenkte die Objekte der Senckenbergischen Naturforschenden Gesellschaft. Heute befindet sich die Sammlung im Museum der Weltkulturen der Stadt Frankfurt a.M. und gehört zu den ältesten Beständen des Museums. Im Rahmen einer Magisterarbeit wurde die Sammlung im Jahr 2000 dokumentiert (Reiß 2000).

$\mathrm{Zu}$ welchem Zeitpunkt die Sammlung nach Europa geschickt wurde und inwieweit es dem Wunsch Wrangells entsprach, dass sie nach Frankfurt gelangte, ist nicht bekannt (Reiß 2000). Die Sammlung umfasste ursprünglich 51 Objekte, von denen neun heute nicht mehr vorhanden sind, da sie größtenteils während des 2. Weltkriegs verloren gingen. Auch wenn nicht eindeutig geklärt ist, wie die Objekte nach Deutschland gelangten, so zeigt sich doch, dass es offenbar möglich war, über private bzw. dynastische halboffizielle Kanäle Gegenstände abzuzweigen und ins Ausland zu senden. Zumindest scheint dies gängige Praxis gewesen zu sein, wie die Sammlungen von Kuprejanov und Etholén zeigen.

\section{Sammlung Kuprejanov}

\section{Landesmuseum Natur und Mensch, Oldenburg}

Im Jahr 1835 gab der Großherzog von Oldenburg, Paul Friedrich August, seinem Oberkammerherrn Alexander Edler von Rennenkampff den Auftrag, ein Naturhistorisches Museum einzurichten. Als Grundstock sollte er die Sammlung des „Kreisphysikus" Dr. med O.E. Oppermann in Delmenhorst erwerben (Hartung 1961:76). Der Ankauf dieser naturkundlichen Sammlung war für die Ausstellung in der Öffentlichkeit und die Belehrung der Bevölkerung gedacht, entsprang also nicht einer privaten Liebhaberei des Großherzogs. Schon 1836 wurden Räumlichkeiten angemietet,

Federgürtel. Pomo, Kalifornien - um 1830. Sammlung Kuprejanov, Landesmuseum Natur und Mensch, Oldenburg.

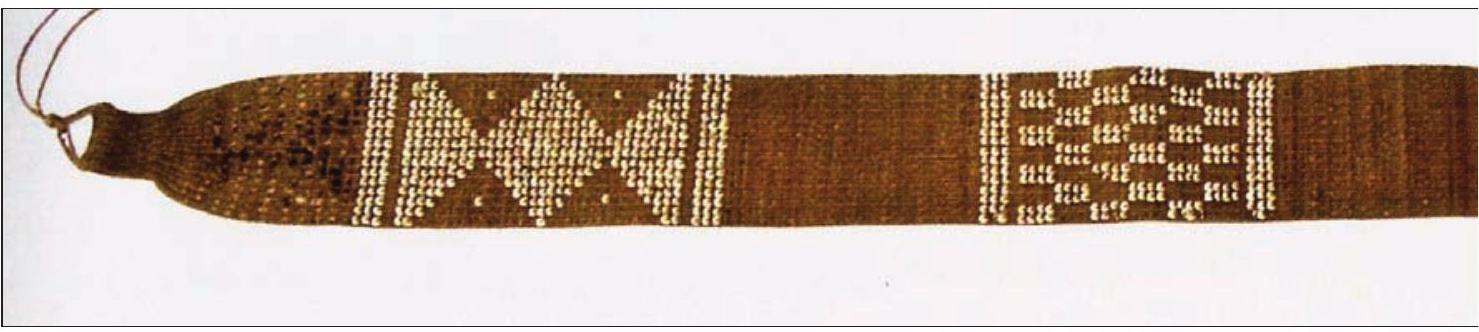


um die Sammlung dem interessierten Publikum zeigen zu können. Ab 1837 gab es eine hauptamtliche Stelle eines Kustos, damit auch eigene wissenschaftliche Arbeit geleistet werden konnte. Erster Kustos wurde Carl Friedrich Wiepken, der dieses Amt bis 1894 ausübte. Im Jahr 1879 zog das Museum in ein eigens errichtetes Gebäude, in dem es sich bis heute befindet. Es heißt derzeit Landesmuseum für Natur und Mensch. Die Sammlung Kuprejanov ist eine der frühesten geschlossenen Sammlungen im Bestand des Museums, da sie schon 1841 in Oldenburg eintraf.

Auf den ersten Blick scheint die Sammlung Kuprejanov gut dokumentiert, da ihr ein an Rennenkampff gerichtetes Begleitschreiben sowie eine Liste der Objekte beigegeben wurden. Beide sind in französischer Sprache abgefasst. Der Brief wurde in St. Petersburg von einem gewissen Grafen Tolstoj unterschrieben, dessen Identität vorläufig noch nicht geklärt ist, der aber offenbar persönlich mit Alexander von Rennenkampff bekannt war. Mehrere Träger dieses Namens hatten zur fraglichen Zeit Positionen inne, die es ihnen ermöglicht hätten, die Sammlung nach Oldenburg zu schicken.

Offenbar war jener Tolstoj für Prinz Peter von Oldenburg tätig, der wiederum in russischen Diensten stand. Tolstoj schreibt, dass der Oberst der Russischen Marine „Kouprianoff“ von seiner Expedition rund um die Welt zurückgekehrt sei und dem gnädigen Herrn verschiedene Objekte verehre, die er aus den russisch-amerikanischen Kolonien mitgebracht habe. Es handele sich dabei um Kleidung, Waffen, Werkzeuge, Boote und Pfeile für die Waljagd. Außerdem enthalte die Sendung ebenfalls Skelette und Bälge von Robben und Vögeln. Tolstoj bemüht sich, von Rennenkampff mit zusätzlichen Informationen zur Sammlung zu versorgen. Er schreibt:

Nun, werter Baron, das sind die einzigen Hinweise, die ich Ihnen geben kann, und um sich selbst weiter zu informieren, füge ich ein Werk eines Obersten bei, der die Welt umrundet und sich dort mehrere Jahre aufgehalten hat. Sein Werk ist nur wenig zufriedenstellend, aber dennoch kann es eine richtige Idee über den Gebrauch ihrer Waffen und über einige der Gegenstände, die ich Ihnen schicke, vermitteln. ${ }^{1}$ (übersetzt von G.B.)

Bislang wurde in Oldenburg noch keine Spur dieses Schriftstücks gefunden. Es ist ebenfalls nicht bekannt, welchen Autor Tolstoj meint. Auch hier kommen mehrere 


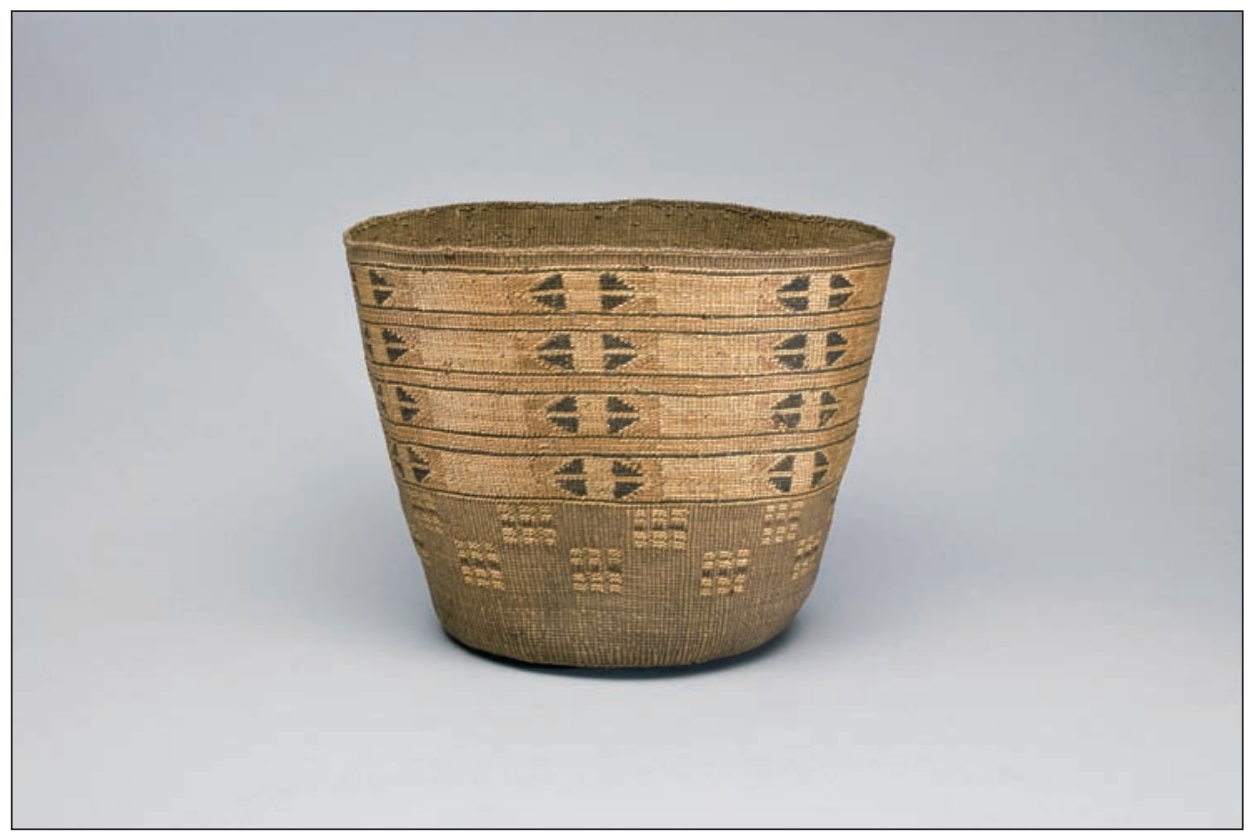

Korb. Tlingit, Alaska - um 1830. Sammlung Kuprejanov. Landesmuseum Natur und Mensch.

Foto: W. Kehmeier.

Marineangehörige in Frage, die Berichte über ihre Aufenthalte in Russisch-Amerika geschrieben haben. Weiterhin enthält der Brief Tolstojs einen Hinweis darauf, dass die Sendung legal und offiziell genehmigt das Land verlassen hat, da er schreibt, er werde eventuelle Fragen bezüglich der Gegenstände an das Kaiserliche Museum weiterleiten, das im übrigen eine noch umfangreichere Sammlung von Kuprejanov erhalten habe. Darüber hinaus erfahren wir im Brief des Grafen, dass die Gegenstände sorgfältig in vier Pakete verpackt mit dem Lübecker Dampfboot Alexandra Russland verlassen werden. Die Sendung ging an Seine Königliche Hoheit Ihre Eminenz den Großherzog zur Weitergabe an Herrn Baron von „Renenkampf“. Dem Brief beigegeben war eine Liste, ebenfalls in französischer Sprache abgefasst: „Katalog der verschiedenen Gegenstände, die von den Einwohnern der Aleuten hergestellt wurden und von Colonel Kouprianoff von seiner Reise um die Welt mitgebracht wurden" (übersetzt von G.B.). ${ }^{2}$

Diese Liste umfasst 89 Nummern, insgesamt 137 Objekte. Von diesen sind, laut Liste, elf Skelette von Meeresvögeln, vier Skelette bzw. Knochen von Säugetieren und 24 ausgestopfte Vogelpräparate. Zieht man die Granate, vulkanische Schlacke, und ein spezielles Moos - aus dem die Aleuten gelbe Farbe gewannen - ab, bleiben 98 ethnographische Objekte. Von diesen 98 ethnographischen Objekten lassen sich bislang nur 31 sicher den aktuellen Inventarnummern des Oldenburger Museums zuordnen. 
$\mathrm{Zu}$ Beginn der 1990er Jahre führte Hermann Devermann eine Gesamtinventur des völkerkundlichen Bestands des Museums durch, der ca. 3500 Objekte aus verschiedenen Regionen umfasst. In den vorhandenen Inventarbüchern, auf die Devermann sich stützte, werden insgesamt 139 Objekte der Sammlung Kurpejanov zugeordnet. Vorerst lässt sich nicht nachvollziehen, auf welcher Grundlage die Zuordnung zur Sammlung Kuprejanov damals erfolgte. Im Einzelnen wird also zu hinterfragen sein, ob es sich wirklich bei all diesen Gegenständen um Objekte aus der Sammlung Kuprejanov handelt. Dazu ist einerseits nötig, einen genaueren Blick auf die Person des Sammlers und seine Reisetätigkeit bzw. seine Kontakte zu anderen potentiellen Sammlern zu werfen, andererseits sind Vergleichsstudien an den Sammlungen in St. Petersburg durchzuführen. Auch die Beziehungen des Grafen Tolstoj zu Kuprejanov einerseits und zu Prinz Peter von Oldenburg und Baron von Rennenkampff andererseits sind eingehender zu beleuchten.

Ivan Antonovič Kuprejanov wurde vermutlich 1799 geboren und trat als 10-Jähriger in die Kadettenschule in St. Petersburg ein. Ab 1819 nahm er an mehreren Weltumsegelungen teil, die ihn in die Antarktis, den Südpazifik und auch nach RussischAmerika führten. So erreichte er beispielweise am 3. September 1823 erstmals Sitka, den Regierungssitz von Russisch-Amerika. Er setzte seine Karriere in der russischen Marine fort und übernahm Aufträge, die ihn nach Finnland, aufs Schwarze Meer und

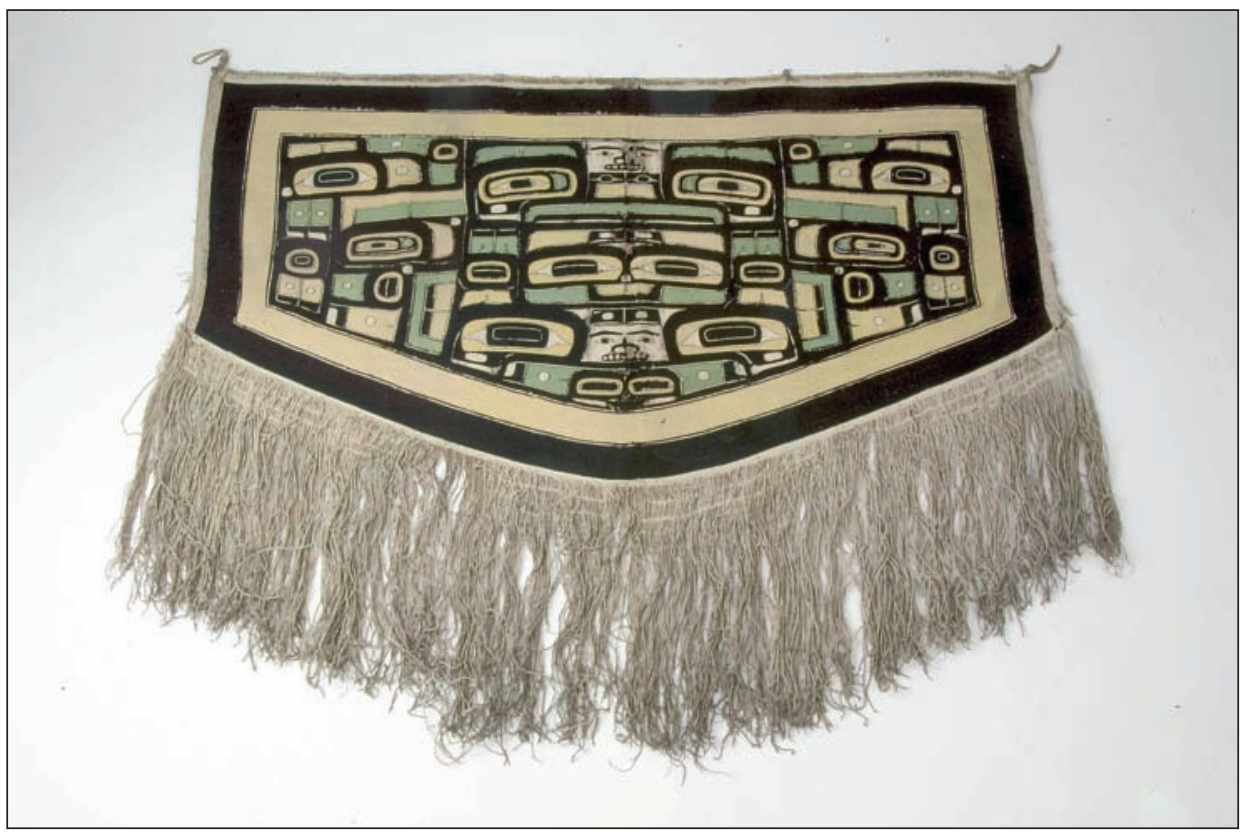

Chilkat-Decke. Tlingit, Alaska - um 1830. Sammlung Kuprejanov. Landesmuseum Natur und Mensch. Foto: W. Kehmeier. 
die Ostsee führten, bis er am 29. August 1834 zum Gouverneur von Russisch-Amerika berufen wurde. Gemeinsam mit seiner Frau Julia Ivanovna kam er am 25. Oktober 1835 in Sitka an und löste Ferdinand von Wrangell ab. Der Posten des Gouverneurs wurde jeweils für fünf Jahre besetzt. Während seiner Dienstzeit in Russisch-Amerika unternahm Kuprejanov mehrere ausgedehnte Reisen, 1836 besuchte er die Insel Kodiak und 1837 die aleutischen Inseln Atka und Unalashka. 1837 schließlich reiste er zu dem befestigten Posten St. Dionysius in der Nähe der Mündung des Stikine und besuchte die Yakutat Bucht. Außerdem beauftragte er auch andere Mitarbeiter der Russisch-Amerikanischen Kompanie, die Region zu besuchen, zu erforschen und zu dokumentieren. Während seiner Zeit als Gouverneur wütete eine Pockenepidemie, die die indigene Bevölkerung drastisch dezimierte und bei der Russisch-Amerikanischen Kompanie zu akutem Arbeitskräftemangel führte (vgl. Luehrmann 2008). Am 1. Mai 1840 wurde Kuprejanov von seinem Nachfolger Arvid Adolf Etholén abgelöst. Er hielt sich aber noch bis zum 30. September in der Kolonie auf, bis er auf dem Seeweg nach St. Petersburg zurückkehren konnte. Über San Francisco, Valparaiso, Kap Hoorn und Rio de Janeiro erreichte er Kronstadt am 13. Juni 1841. Bereits im Oktober desselben Jahres wurden die Objekte von St. Petersburg nach Oldenburg geschickt. In der Folge befehligte Kuprejanov Fregatten auf der Ostsee und wurde im Oktober 1852 zum Vizeadmiral ernannt. Er starb am 30. April 1857 und wurde auf dem Orthodoxen Smolensker Friedhof in St. Petersburg beigesetzt.

Kuprejanovs Nachfolger Arvid Adolf Etholén hatte nach seiner Ausbildung in der Marineakademie in St. Petersburg bereits zehn Jahre in Russisch-Amerika in unterschiedlichen Positionen gearbeitet, bevor er Gouverneur wurde. Eine Zeitlang war er als Assistent Ferdinand von Wrangells tätig gewesen. Er war sehr viel gereist und hatte somit alle russischen Stützpunkte in Amerika besucht.

Wrangell und Etholén hatten sich bereits während der Weltumsegelung unter Golovnin (1817-1819) kennen gelernt. Im Jahr 1824 reiste Etholén auf jenem Schiff zurück nach Kronstadt, auf dem auch Kuprejanov Dienst tat. Es ist also davon auszugehen, dass alle drei recht gut miteinander bekannt waren. Wahrscheinlich ist es kein Zufall, dass ausgerechnet diese drei Gouverneure reichhaltige Sammlungen auch ins Ausland schickten. Gemeinsam ist den drei Sammlungen, dass sie neben Objekten der Aleuten und Alutiiq auch solche aus Kalifornien beinhalten, da sowohl Wrangell als auch Kuprejanov und Etholén in Fort Ross und San Francisco gewesen waren. Etholén hatte bereits von seinen früheren Aufenthalten Sammlungen nach Finnland geschickt. Diese kamen 1825 und 1826 in Turku an, gingen aber während eines großen Brandes in Turku im Jahr 1827 verloren.

Die Entstehung dieser drei Sammlungen fällt in die Zeit, in der die Kaiserliche Akademie der Wissenschaften in St. Petersburg großes Interesse an der naturräumlichen Ausstattung von Russisch-Amerika zeigte. So wurde beispielsweise Il'ja Gavrilovič Voznesenskij auf Kosten der Akademie für drei Jahre nach Russisch-Amerika geschickt, ausschließlich, um naturkundliche Sammlungen anzulegen. Sein Vertrag 


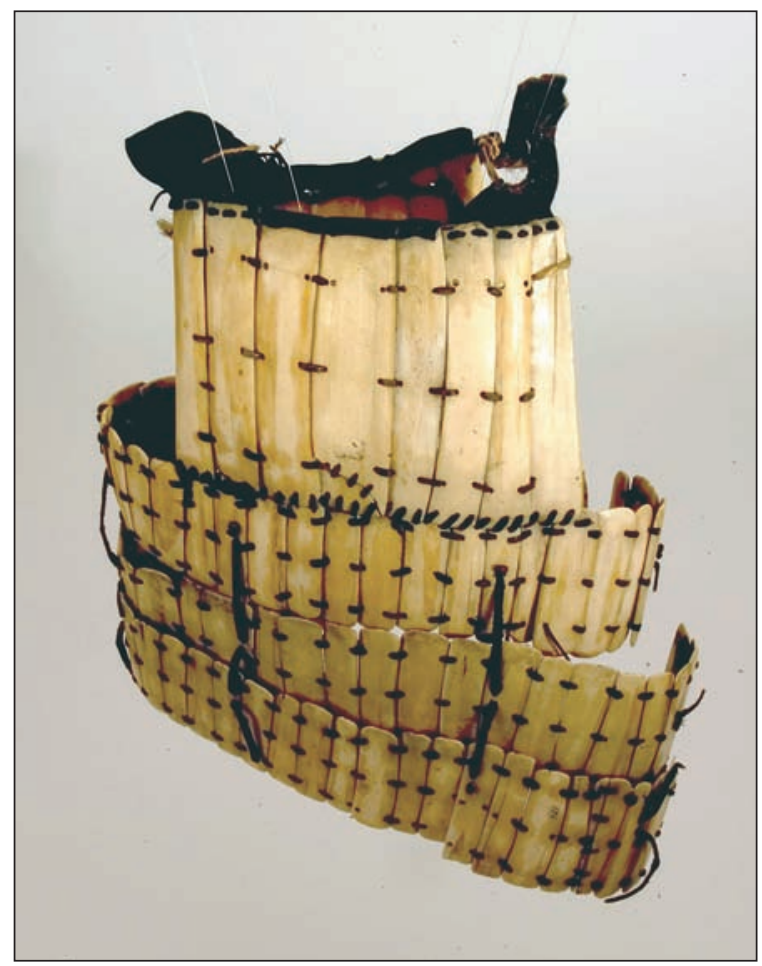

Lamellenpanzer aus Walroßrippen. Inupiaq, Alaska - um 1830. Landesmuseum Natur und Mensch.

Foto: W. Kehmeier.

wurde zunächst auf fünf, dann auf zehn Jahre verlängert. Voznesenskij reiste auf demselben Schiff wie Etholén, so dass beide gleichzeitig am 1. Mai 1840 in Sitka eintrafen. Kuprejanov, der gerade noch im Amt war, als Voznesenskij ankam, unterstützte dessen Arbeit, indem er ihm einen talentierten Gehilfen zur Seite stellte und ihm gestattete, auf Kompanieschiffen zu reisen. Später reiste Voznesenskij gemeinsam mit Etholén. Neben naturkundlichen Sammlungen legte er auch umfangreiche ethnographische Sammlungen an, die sich im Museum für Anthropologie und Ethnographie Peter der Große in St. Petersburg befinden. Nach seiner Rückkehr wurde Voznesenskij Kurator im Zoologischen Museum.

Die Sammlung Kuprejanov gehört zu jenen Sammlungen, die in Fachkreisen gut bekannt sind. Das Landesmuseum Natur und Mensch erhält häufig Anfragen bzw. Leihersuchen für einige der besonderen Objekte aus der Sammlung. Mehrere Versuche der wissenschaftlichen Bearbeitung und Veröffentlichung der Sammlung scheiterten an den finanziellen Mitteln. Um so wünschenswerter wäre es, die Sammlung Kuprejanov in vergleichbarer Form wie die Sammlungen Cook/Forster und Asch zu publizieren. Dank der Initiative von Brigitta Hauser-Schäublin kann derzeit wieder an der Samm- 
lung gearbeitet werden. Eine Publikation der Sammlung wäre nicht zuletzt vor dem Hintergrund sinnvoll, dass auch in den Museen Alaskas reges Interesse an den Objekten besteht und zwar nicht in Form von Rückgabeforderungen. Es wird lediglich der Wunsch nach Zugänglichkeit geäußert, insbesondere wie König (1993:63f.) schreibt: „[...], um die Techniken ihrer Vorfahren wieder erlernen, anwenden und eigene Handwerker und Künstler ausbilden zu können.“

An den Sammlungen aus Russisch-Amerika zeigt sich beispielhaft, dass Ethnographica „vom Ende der Welt“ auch jenseits des kolonialen Kontextes reges Interesse fanden. Wurden sie in Russland als Dokumente aus der fernen Kolonie gesehen, so entwickelten sie sich in Europa zu wertvollen Geschenken, die dazu eingesetzt wurden, verwandtschaftliche Beziehungen zu pllegen oder sonstigen Verpflichtungen nachzukommen. Wie im eingangs aufgeführten Zitat von Brigitta Hauser-Schäublin formuliert, verändert sich unsere Sichtweise mit jeder neuen Epoche. Phasen besonderen Interesses an den Objekten wechselten mit relativem Desinteresse. Aus heutigem Blickwinkel wird ihnen aufgrund des vergleichsweise hohen Alters besonderer Wert für Studien zum Kulturwandel beigemessen, andererseits werden hervorragende Einzelstücke als Kunstwerke geschätzt und entsprechend präsentiert. Und auch für die Angehörigen der Kulturen, aus denen die Artefakte stammen, hat sich die Bedeutung der Objekte gewandelt. Von alltäglichen Gebrauchsgegenständen wurden sie zu Zeugen der eigenen Geschichte und dienen als Inspiration für aktuelle Künstler und Handwerker.

\section{Anmerkungen}

1 „[...] et afin de Vous mettre à même de prendre quelques Reinseignements j’y joins un ouvrage d'un Colonel qui a fait le tour du Monde, et qui a fait un Séjour de quelques années; Son Ouvrage est bien peu satisfaisant, mais pourtant peut donner une idee Juste Sur la manière de se Servir de leurs armes et sur plusieurs articles que je Vous envoi [...]" (Brief des Grafen Tolstoj an Baron Rennenkampff, Museum für Natur und Mensch Oldenburg).

2 „Catalogue de diverses productions de l'industrie des Habitants des Iles Aléoutes, apportées par le colonel Kouprianoff dans son voyage autour du monde."

\section{Literatur}

Amburger, Erik (1966) Geschichte der Behördenorganisation Russlands von Peter dem Großen bis 1917. Leiden: E.J. Brill.

Black, Lydia T. (2004) Russians in Alaska 1732-1867. Fairbanks, Alaska: University of Alaska Press.

Bucher, Gudrun (2007) Ethnographic Objects - Russian America. In: Brigitta Hauser-Schäublin und Gundolf Krüger (Hg.) Sibirien und Russisch-Amerika: Kultur 
und Kunst des 18. Jahrhunderts. Die Sammlung von Asch - Göttingen, S. 243-252. München: Prestel.

Feest, Christian (1993) European Collecting of American Indian Artefacts and Art. Journal of the History of Collections 5(1):1-11.

Hartung, Wolfgang (1961) 125 Jahre staatliches Museum für Naturkunde und Vorgeschichte in Oldenburg. Museumskunde 30(2):76-86.

Hauser-Schäublin (1998) Getauschter Wert - Die verschlungenen Pfade der Objekte. In: Brigitta Hauser-Schäublin und Gundolf Krüger (Hg.): James Cook - Gaben und Schätze aus der Südsee, S. 11-29. München: Prestel.

Hauser-Schäublin, Brigitta und Gundolf Krüger (Hg.) (1998)James Cook - Gaben und Schätze aus der Südsee. München: Prestel.

Hauser-Schäublin, Brigitta und Gundolf Krüger (Hg.) (2007) Siberia and Russian America: Culture and Art from the 1700s - The Asch Collection, Göttingen; Sibirien und Russisch-Amerika: Kultur und Kunst des 18. Jahrhunderts - Die Sammlung von Asch, Göttingen. München: Prestel.

König, Viola (1993) Auf den Spuren deutscher Entdecker und Forscher in Russisch Amerika. Alaska und die Nordwestküste im Spiegel alter völkerkundlicher Sammlungen in Bremen und Niedersachsen. In: TenDenZen - Jabrbuch des ÜberseeMuseums 2:27-66.

Luehrmann, Sonja (2008) Alutiiq Villages under Russian and U.S. Rule. Fairbanks, Alaska: University of Alaska Press.

Pierce, Richard A. (1990) Russian America: A Biographical Dictionary. Kingston, Ontario: Limestone Press (Alaska History 33).

Reiß, Barbara Johanna (2000) Die Alaska Sammlung des Barons Ferdinand P. von Wrangell im Museum für Völkerkunde Frankfurt. Magisterarbeit, Universität Frankfurt.

Rousselot, Jean-Loup (1994) Kanuitpit? Kunst und Kulturen der Eskimo - Eine Auswabl aus den Museumssammlungen. München: Staatliches Museum für Völkerkunde.

Rousselot, Jean-Loup (2007) Russisch Amerika - Die frühe nordamerikanische Sammlung. In: Claudius Müller und Wolfgang Stein (Hg.): Exotische Welten. Aus den völkerkundlichen Sammlungen der Wittelsbacher 1806-1848, S.71-80. Dettelbach: Röll.

Urban, Manfred (1998) Die Erwerbungsgeschichte der Göttinger Sammlung. In: Brigitta Hauser-Schäublin und Gundolf Krüger (Hg.): James Cook - Gaben und Schätze aus der Südsee, S. 56-85. München: Prestel. 